\title{
FORMACIÓN INICIAL EN INCLUSIÓN EN LOS GRADOS DE MAESTRO EN EDUCACIÓN PRIMARIA
}

\section{Initial Training in Inclusion in the Teacher Degrees in Primary Education}

Joan Jordi MunTANER-GuASP Universitat de les Illes Balears joanjordi.muntaner@uib.es

Bartomeu MuT-AmenguAL Universitat de les Illes Balears

Carme Pinya-Medina Universitat de les Illes Balears

Recepción: 13 de abril de 2020 Aceptación: 4 de marzo de 2021

RESUMEN: La presente investigación se centra en la descripción y el análisis de la formación inicial que se ofrece en las universidades públicas españolas en el grado de Maestro en Educación Primaria con relación a la educación inclusiva. Para ello optamos por un diseño cualitativo de corte descriptivo interpretativo a partir del estudio de los planes de estudio del grado de Maestro en Educación primaria para el año académico 2019-2020, en referencia tanto a las asignaturas comunes como obligatorias. Los resultados muestran que la oferta formativa se mantiene, en la mayoría de casos, anclada en el paradigma deficitario y/o integrador, tanto en relación con las asignaturas comunes para todos los estudiantes, que siguen centradas en la categorización del alumnado y en buscar propuestas especiales para alumnos especiales, como en los títulos y asignaturas que componen las menciones relativas a la formación del profesorado de apoyo. Son muy limitadas las asignaturas, títulos y propuestas que puedan enmarcarse en el paradigma de una educación para todos, siguiendo los principios y fundamentos de la investigación actual en educación. Podemos concluir que la formación inicial de Maestros en Educación Primaria en el ámbito de la educación inclusiva es clara y significativamente insuficiente e incoherente con los conocimientos científicos actuales. 
Palabras Clave: educación inclusiva; educación superior; formación inicial; formación de maestros.

ABSTRACT: This research focuses on the description and analysis of the initial training offered in Spanish public universities in the degree of Master in Primary Education related to Inclusive Education. For this we opt for a qualitative design of interpretative descriptive cut from the study of the web pages where the curricula of the Master's degree in Primary Education for the academic year 2019-2020 are specified, in reference to both the common and compulsory subjects for all students of the degree as in the title and the training proposal offered in the mention of the field of inclusive education. The results show that the training offer is maintained in most cases anchored in the deficit and / or integrative paradigm, both in relation to common subjects, which remain focused on the categorization of students and in seeking special proposals for special students. The subjects, titles and proposals that can be framed in the paradigm of education for all are very limited, following the principles and foundations of current research in education. We can conclude that the initial training of Teachers in Primary Education in the field of Inclusive Education is clearly and significantly insufficient and inconsistent with current scientific knowledge.

KEYwORDS: inclusive education; higher education; initial teacher training.

\section{Introducción}

$\mathrm{E}$ L DERECHO A UNA EDUCACIÓN DE CALIDAD para todos los niños y niñas, sean cuales sean sus características, sin exclusiones ni discriminación, se enmarca hoy en el paradigma de la educación inclusiva, que tiene como objetivo plantear un modelo educativo de éxito para todos.

El derecho a una educación inclusiva está reconocido y avalado por organismos y entidades como Unesco (1994), ONU (2006), AEDEE (2003) y OCDE (2012), que la promueven claramente en todas sus directrices internacionales. Así, en la Declaración de Incheon, ciudad de Corea donde se celebró el Foro Mundial sobre la Educación 2015 y que concluyó con la aprobación de la Declaración de Incheon para la Educación 2030 (Unesco, 2015), se plantean dos ideas fundamentales: la educación inclusiva, que reconoce a todos los niños y jóvenes como sujetos activos y competentes, demandando la igualdad de oportunidades en términos de acceso a las experiencias educativas, al respeto a sus diferencias individuales y a la calidad de la educación, poniendo el énfasis en sus logros y metas y no en sus dificultades. Paralelamente, la justicia social, la inclusión social y la educación inclusiva aparecen vinculadas e indisociables, como componentes para aspirar a una sociedad inclusiva, que constituye el fundamento mismo del desarrollo social sostenible.

Estos principios y claves del paradigma de la educación inclusiva se encuentran presentes en la legislación educativa española. Como contempla Chiner (2018), se establecen medidas de atención a la diversidad que tienen como objetivo garantizar la educación de todas y todos los alumnos y plantean la educación inclusiva como un 
objetivo a alcanzar en todos los centros y aulas del país, aunque el nivel de aplicación en la realidad de este modelo educativo esté hoy muy alejado de esas intenciones.

La aplicación de los principios y fundamentos de la educación inclusiva a la realidad de los centros educativos requiere la implicación de todo el profesorado, que debe presentar unas actitudes, conocimientos y habilidades coherentes con estos planteamientos. Así, conocer y analizar la formación inicial que las universidades ofrecen a los futuros maestros de Educación Primaria en cuanto al paradigma de la educación inclusiva es una propuesta relevante y fundamental para el desarrollo y la realización de este modelo educativo.

\subsection{La formación inicial en Educación Primaria}

La formación inicial de los maestros y maestras de Educación Primaria en España se rige, actualmente, por los principios y normas generales del Espacio Europeo de Educación Superior, que se concretan en el caso que nos ocupa por la orden ECI/3857/2007 (BOE, 2007), donde se establecen los requisitos para la verificación de los títulos universitarios oficiales que habilitan para el ejercicio de la profesión de maestro en Educación Primaria. En esta orden se especifican las competencias, tanto generales como específicas, por desarrollar a lo largo del plan de estudios.

Los planes de estudio de las enseñanzas universitarias oficiales del grado de Maestro en Educación Primaria tienen una carga lectiva de 240 créditos ECTS, que se dividen en asignaturas básicas, obligatorias y optativas, además de los créditos correspondientes al prácticum y al trabajo final de grado. Las asignaturas optativas pueden agruparse proponiendo menciones calificadoras, entre 30 y 60 créditos ECTS, adecuadas a los objetivos, ciclos y áreas de la Educación Primaria, así como aquellas asignaturas que capaciten para el desarrollo de actividades asociadas a las competencias como biblioteca escolar, las tecnologías de la información y la comunicación y la educación de personas adultas (BOE, 2007).

Entre las competencias que debe adquirir el alumnado, futuros maestros y maestras de Educación Primaria, se incluye la de diseñar y regular espacios de aprendizaje en contextos de diversidad y que atiendan a la igualdad de género, a la equidad y al respeto a los derechos humanos que conformen los valores de la formación ciudadana (BOE, 2007), lo cual marca las bases de la educación inclusiva, puesto que, como afirma Alba (2017, p. 12): "se trata de organizar, planificar, enseñar y formar al profesorado partiendo de la realidad en la que existe la diversidad".

Interpretar la diversidad como un hecho natural e incuestionable, que afecta a todo el alumnado sin excepciones, se convierte en una tarea y responsabilidad de todo el profesorado, que requiere formación en este ámbito. Echeita (2012, p. 12) afirma: "Resaltar y reforzar el mensaje fundamental de que tales competencias son y serán necesarias para todo el profesorado, en consonancia con el principio básico de que la educación inclusiva hace referencia a la educación de todo el alumnado y no sólo de algunos". 
La formación inicial del profesorado, por tanto, debe cubrir la necesidad de contar con un profesorado cualificado para abordar con éxito una educación para todos y todas, puesto que la educación inclusiva no es un asunto marginal del sistema educativo, sino esencial al mismo, en tanto en cuanto nos interpela sobre el proyecto de sociedad que se quiere ir construyendo (Unesco, 2008). Esta formación inicial debe contemplar tanto conocimientos y habilidades, relativos a la atención ordinaria y natural de la diversidad de todo el alumnado, como centrarse en las actitudes y valores que se hallan en la base de la educación inclusiva.

La aplicación real de los principios y valores de la educación inclusiva en todas las aulas y centros educativos de todo el sistema educativo requiere la formación de todo el profesorado, puesto que depende en gran medida de su preparación, de sus competencias y actitudes para enseñar en un entorno donde la diversidad es una realidad ineludible (González-Gil et al., 2017). Coherente con este planteamiento la European Agency for Development un Special Needs (2011) identifica cuatro valores fundamentales, vinculados a la enseñanza y el aprendizaje, básicos para la adquisición de competencias para trabajar en educación inclusiva: a) respetar la diversidad del alumnado. b) favorecer a todo el alumnado, c) trabajar en equipo y d) promover el desarrollo personal y profesional permanente.

Por otra parte, debemos señalar que la incorporación del trabajo en competencias inclusivas para todo el profesorado no está en contradicción -señala Echeita (2012)con una formación especializada en ámbitos relacionados con el alumnado que se considera de necesidades educativas especiales, por ello es importante conocer y analizar tanto la formación general en educación inclusiva que se ofrece a quien se gradúa en Maestro de Educación Primaria, como la formación específica y en profundidad que se plantea para el alumnado que escoge la mención de apoyo educativo, que tiene nomenclaturas diversas y dispares según cada universidad.

Se trata de dos formaciones complementarias, que no contradicen el espíritu, los valores ni el paradigma de la escuela inclusiva: ofrecemos una formación para todos los maestros, que nos permita construir y desarrollar una escuela para todos, que atienda a la diversidad desde la equidad y la calidad educativa, $y$, a la vez, se prepara de manera específica a parte del alumnado para desempeñar funciones de apoyo y soporte al maestro/a tutor o especialista para completar y favorecer una actuación conjunta, compartida y en equipo para atender a todo el grupo clase sin excepciones ni discriminaciones (Torres y Castillo, 2016).

\subsection{La educación inclusiva}

Alcanzar una educación de calidad para todos y con todos es un objetivo común y compartido por la totalidad de los ámbitos relacionados con la educación en el mundo entero. Este movimiento se sitúa, a nivel pedagógico y educativo, en torno al marco aglutinador conocido como educación inclusiva, la cual pretende acabar con la exclusión, discriminación o segregación de cualquier alumno o alumna, sea porque no tienen posibilidad de acceder a la educación reglada, sea porque tienen situaciones 
de clara desventaja o vulnerabilidad dentro del propio sistema educativo sean cuales sean sus características.

Este planteamiento nos introduce en un contexto de cambio, de innovación y de mejora del sistema educativo, que surge desde el cuestionamiento de las modalidades de trabajo existentes y dominantes en la actualidad. En la práctica, ese contexto de cambio se concreta en la identificación y eliminación de las barreras al aprendizaje y la participación con el objetivo de maximizar los facilitadores que apoyen y posibiliten el desarrollo de este proceso hacia la inclusividad (Booth y Ainscow, 2002). Este modelo educativo inclusivo es otra manera de entender y actuar en el contexto social y educativo; significa un cambio de paradigma, de perspectiva y de referentes para entender y analizar la realidad desde el foco de la justicia social y los derechos humanos.

La educación inclusiva se fundamenta en tres antecedentes básicos:

a) La interpretación de la diferencia. Las diferencias entre las personas son un hecho natural e incuestionable, pues hay muchas características que nos hacen únicos e irrepetibles. Si la diferencia entre las personas es inevitable, la cuestión clave se centra en determinar cómo interpretamos esta diferencia, pues, como escriben Thomas y Laxley (2007, p. 135): "que la diferencia se interprete de forma positiva, como diversidad; o negativa, como desviación o déficit, depende del pensamiento de la persona o grupo de personas que observan esta definición”. De esta posición dependerá la respuesta que se plantee: si la interpretamos como una realidad negativa, como un problema que debemos resolver, como un déficit o una desviación, la propuesta de actuación nos conduce a la segregación o a la discriminación. Al contrario, si entendemos que la diversidad es un aspecto natural y enriquecedor, nos situamos en una perspectiva positiva en la que no hay categorías, que nos obliga a aprender a trabajar y a convivir con ella.

b) El enfoque en el individuo o en el contexto. La realidad puede observarse desde dos posiciones antagónicas: fijarse en los problemas de la persona vulnerable, en sus déficits, limitaciones o características individuales; o bien, analizarla como una realidad social, basada en las condiciones que ofrece el entorno al desarrollo y participación de las personas, sean cuales sean sus características, capacidades o actitudes, en su hábitat natural.

c) Creencias, valores y criterios. Cada persona fundamenta sus decisiones en sus creencias, valores o criterios como lente a través de la cual analiza la realidad circundante. Si no se produce un cambio radical en estos referentes básicos, siempre se encuentran excusas, razones y motivos para no cambiar, y, en consecuencia, no avanzar. Se trata de construir una nueva cultura que reconozca las diferencias como un hecho natural y no las oprima o discrimine. Oliver (1998, p. 54) lo explica utilizando la metáfora del juego: "Hay enfoques que se preocupan por permitir que los grupos excluidos se integren en el juego tal y como es, mientras que los planteamientos emancipadores se preocupan tanto por conceptualizar como por crear un juego nuevo, donde nadie sea excluido por adelantado". 
La educación inclusiva es un proceso de cambio, desde la reflexión y la convicción de la necesidad de ofrecer a la comunidad educativa un modelo de actuación que permita a todo el alumnado compartir y aprender juntos en un mismo espacio y tiempo, sin categorizar ni diferenciar por las características individuales, acabando con la exclusión y propiciando el desarrollo de propuestas de calidad válidas para todos. La Unesco (2005, p. 13) la define como: "Un proceso de abordaje y respuesta a la diversidad de las necesidades de todos los alumnos a través de la creciente participación en el aprendizaje, las culturas y las comunidades, y de la reducción de la exclusión dentro y desde la educación. Implica cambios y modificaciones en los enfoques, las estructuras, las estrategias, con una visión que incluye a todos los niños y niñas de la franja etaria adecuada y la convicción de que es responsabilidad del sistema regular educar a todos y todas".

Este modelo de educación no es solo una simple cuestión organizativa, curricular o metodológica, sino una manera distinta de entender y practicar la educación. Estos planteamientos nos sitúan ante los principios claves y fundamentales que determinan las prácticas inclusivas y que podemos concretar en cuatro puntos:
a) La diferencia entre las personas es un hecho natural: no hay categorías de alumnos.
b) La diversidad no puede ser eliminada: actuamos en el contexto, no en la persona.
c) Realizamos siempre agrupamientos heterogéneos.
d) Los apoyos son necesarios y deben ser normalizados en el grupo.

Este modelo educativo inclusivo se aplica en los centros y aulas ordinarios a partir de planteamientos más flexibles y abiertos, basados en la equidad y la calidad para todo el alumnado sin excepciones. Escudero y Martínez (2011, p. 93) lo señalan con claridad: "Una pedagogía rica, estimulante, con variedad de métodos, materiales, flexible, que tome no solo en consideración la variedad, tolerándola, sino que la entienda y la valore como un desafío al que responder, como un recurso valioso".

\section{Método}

Esta investigación se centra en el análisis de la formación en educación inclusiva que ofrecen las universidades públicas españolas en los títulos del grado de Maestro en Educación Primaria, así como en las menciones directamente relacionadas con la inclusión educativa. Se trata de una investigación descriptiva-interpretativa a partir del estudio y análisis de los planes de estudio publicados para el curso 2019-2020 en las páginas web de cada universidad.

\subsection{Objetivos}

Se plantean dos objetivos:

a) Conocer y analizar las asignaturas tanto de formación básica como obligatorias del título del grado de Maestro en Educación Primaria, que ofrecen las uni- 
versidades públicas españolas, directamente relacionadas con la educación del alumnado vulnerable. No se recogen ni analizan aquellas asignaturas que pudieran tener algún tema o apartado relacionado con la formación y atención del alumnado vulnerable, como pueden ser Psicología de la Educación, Didáctica General u Orientación.

b) Conocer y analizar las menciones ofrecidas en los títulos del grado de Maestro en Educación Primaria que se refieren de manera específica a la educación del alumnado más vulnerable en el marco de la educación inclusiva.

\subsection{Diseño}

Para la consecución de los objetivos señalados optamos por un diseño cualitativo de corte descriptivo-interpretativo. Como técnica de investigación se emplea el análisis documental y de contenido centrado en los planes de estudio del grado de Maestro en Educación Primaria ofertados en las universidades públicas españolas en el año académico 2019-2020.

\subsection{Población y muestra}

Se han recogido y estudiado los planes de estudio de las 39 universidades públicas españolas que ofrecen el título de grado en Maestro de Educación Primaria en el año académico 2019-2020. Estas universidades se distribuyen geográficamente según la Tabla 1. Por otra parte, señalar que algunas de estas universidades ofrecen este grado en distintas localidades y pueden presentar algunas diferencias en su plan de estudios, para la realización de esta investigación solamente se ha utilizado un único plan de estudios para cada universidad.

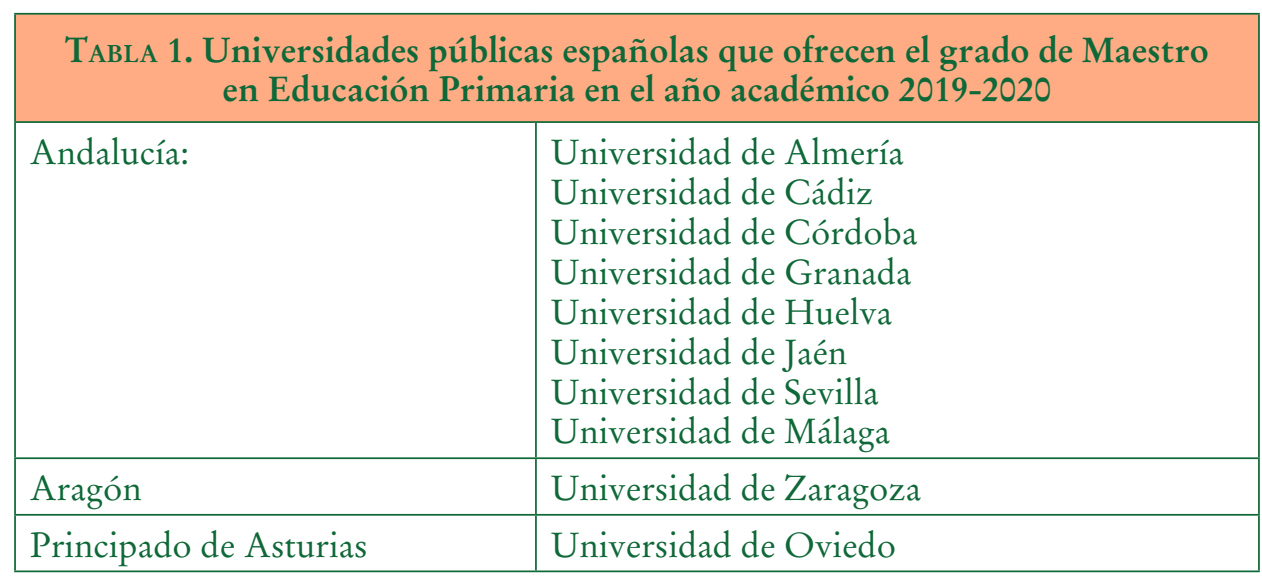

Ediciones Universidad de Salamanca / CC BY-NC-ND

Siglo Cero, vol. 52 (4), 2021, octubre-diciembre, pp. 9-27 


\begin{tabular}{|l|l|}
\hline Illes Balears & Universitat de les Illes Balears \\
\hline Islas Canarias & $\begin{array}{l}\text { Universidad de Gran Canaria } \\
\text { Universidad de La Laguna }\end{array}$ \\
\hline Cantabria & Universidad de Santander \\
\hline Castilla y León & $\begin{array}{l}\text { Universidad de León } \\
\text { Universidad de Burgos } \\
\text { Universidad de Salamanca } \\
\text { Universidad de Valladolid }\end{array}$ \\
\hline Castilla-La Mancha & Universidad de Castilla-La Mancha \\
\hline Cataluña & $\begin{array}{l}\text { Universitat Autónoma de Barcelona } \\
\text { Universitat de Barcelona } \\
\text { Universitat de Girona } \\
\text { Universitat de Lleida } \\
\text { Universitat Rovira i Virgili }\end{array}$ \\
\hline Comunitat Valenciana & $\begin{array}{l}\text { Universitat Jaume I } \\
\text { Universitat d'Alacant } \\
\text { Universitat de València }\end{array}$ \\
\hline Extremadura & Universidad de Extremadura \\
\hline Galicia & $\begin{array}{l}\text { Universidad de Santiago de Compostela } \\
\text { Universidad de A Coruña } \\
\text { Universidad de Vigo }\end{array}$ \\
\hline Comunidad de Madrid & $\begin{array}{l}\text { Universidad de Alcalá } \\
\text { Universidad Complutense } \\
\text { Universidad Autónoma de Madrid } \\
\text { Universidad Juan Carlos }\end{array}$ \\
\hline Región de Murcia & Universidad de Murcia \\
\hline Comunidad Foral Navarra & Universidad Pública de Navarra \\
\hline País Vasco & Universidad del País Vasco \\
\hline La Rioja & Universidad de La Rioja \\
\hline
\end{tabular}

\subsection{Procedimiento de recogida y análisis de datos}

Para la recogida de datos diseñamos una ficha de registro con dos apartados de análisis: 1) asignaturas comunes (número, título, curso, semestres, tipología y contenidos) y 2) menciones específicas (título, créditos de la mención, asignaturas, prácticas, TFG y otros). Cada una de ellas con cinco categorías. De esta forma, pretendemos identificar las referencias claves a la educación y formación del alumnado vulnerable 
que se trabaja en los grados de Maestro en Educación Primaria. El procedimiento desarrollado en la investigación consta de tres fases:

a) Búsqueda y selección de la documentación requerida en las páginas web de cada una de las universidades públicas españolas.

b) Aplicación de la ficha de registro de recogida de datos para cada una de las 39 titulaciones estudiadas.

c) Análisis descriptivo-interpretativo de los datos atendiendo a los objetivos y a las categorías propuestas para cada uno de ellos.

\section{Resultados}

El análisis de la información recogida se realiza a partir de las diferencias encontradas entre los diversos enfoques que suponen formas distintas de enfrentarse a la diversidad y que se reflejan tanto en los títulos de las asignaturas estudiadas como en las menciones objetivo de este trabajo.

Podemos diferenciar tres enfoques teóricos, que suponen formas distintas de interpretar la presencia de la diversidad en las aulas y centros escolares y, en consecuencia, plantean modelos de intervención y se proponen actuaciones docentes diferenciadas. Así lo apuntan Biencinto-López et al. (2009), señalan que atender a la diversidad de los estudiantes supone diferentes formas que van de la exclusión de las diferencias para tratarlas fuera de los grupos ordinarios a la inclusión de las diferencias para tratarlas individualmente en el aula.

A continuación, se muestra la elaboración conceptual a partir de Montobbio (1995) y completada en Muntaner (2009), donde podemos comprobar las diferentes maneras de interpretar y de actuar ante las diferencias según el enfoque teórico utilizado:

a) Modelo Terapéutico: centrado en lo patológico, donde lo relevante es el déficit de la persona sobre la que debemos actuar; muestra un modelo tradicional que conduce a la segregación y a la exclusión de la diferencia.

b) Modelo Integrador: centrado en la discapacidad, se introduce el concepto de necesidades educativas especiales, que quiere dar importancia al contexto, pero en la práctica se entiende como sinónimo del déficit. Es un modelo rehabilitador y asimétrico, que incorpora la diferencia, pero exige la adaptación de esta a las demandas y obligaciones de lo ordinario.

c) Modelo Inclusivo: centrado en la persona, donde lo relevante es el contexto, que debe adaptarse y modificarse para responder a las características de todos y cada uno de los y las estudiantes, por ello se incorpora el concepto de barreras y facilitadores a la participación. Es un modelo global, que ofrece oportunidades y tiene en la educación su argumento y en los apoyos normalizados su manera de actuar.

Objetivo 1: Las asignaturas comunes de formación básica y/o obligatoria, que han de cursar todos los estudiantes del grado de Maestro en Educación Primaria, que tienen en el título alguna referencia a la diversidad de los estudiantes (ya sea a la in- 
tervención, características, educación, psicología de grupos o colectivos vulnerables), se distribuyen en cuanto al número en las 39 universidades públicas españolas, de la siguiente manera:

\begin{tabular}{|c|c|c|}
\hline \multicolumn{2}{|c|}{ TABLA 2. Distribución de las asignaturas obligatorias por universidades } \\
\hline N. $^{\text {o de universidades }}$ & Asignaturas ofrecidas & Total (sumatorio) \\
\hline 19 & 1 & $19(19)$ \\
\hline 9 & 2 & $18(37)$ \\
\hline 3 & 3 & $9(46)$ \\
\hline 1 & 4 & $4(50)$ \\
\hline 3 & $1+(1$ optativa) & $3(53)$ \\
\hline 1 & $2+(2$ optativas $)$ & $2(55)$ \\
\hline 3 & Ninguna & $0(55)$ \\
\hline
\end{tabular}

Todas estas asignaturas comunes, no propias de una mención, tienen una carga lectiva de 6 créditos ECTS y se cursan en primero o segundo curso, ya sea en el primer o segundo semestre.

Si analizamos los títulos de estas 55 asignaturas comunes y/o obligatorias para todos los estudiantes del grado, podemos agruparlas en dos grandes bloques:

a) Aquellas que se centran en el individuo o en sus deficiencias o limitaciones, propias de un paradigma terapéutico y deficitario, de las que contamos 19. Algunos ejemplos son: "Trastornos del aprendizaje y el desarrollo", "Dificultades de aprendizaje en educación primaria", "Psicología de las dificultades del aprendizaje", "Trastornos de la niñez".

b) Aquellas que se centran en la intervención y sitúan el foco de atención en el contexto, propias del paradigma integrador o del inclusivo, que suman 36 . Algunos ejemplos son: "Sociología de la educación", "Interculturalidad e inclusión", "Currículo en contextos diversos", "Teoría y práctica de la educación inclusiva", "Educación inclusiva y respuesta a la diversidad", "Bases de la educación inclusiva".

Si cruzamos la información presentada en los puntos anteriores, los resultados obtenidos se distribuyen de la manera siguiente: 


\begin{tabular}{|c|c|c|c|}
\hline \multicolumn{4}{|c|}{$\begin{array}{c}\text { TABLA 3. Asignaturas comunes centradas } \\
\text { en la deficiencia o en la intervención }\end{array}$} \\
\hline $\begin{array}{c}\text { N. }^{\circ} \text { de } \\
\text { universidades }\end{array}$ & $\begin{array}{c}\text { Número de asignaturas } \\
\text { comunes y obligatorias }\end{array}$ & $\begin{array}{c}\text { Asignaturas } \\
\text { tipo A }\end{array}$ & $\begin{array}{c}\text { Asignaturas } \\
\text { tipo B }\end{array}$ \\
\hline 26 & 1 & 7 & 19 \\
\hline 10 & 2 & 9 & 11 \\
\hline 3 & 3 & 3 & 6 \\
\hline
\end{tabular}

Objetivo 2: Analizar las menciones del grado de Maestro en Educación Primaria que se refieren a la inclusión, el apoyo, la educación especial o la pedagogía terapéutica.

Encontramos una gran diversidad de opciones, que se distribuyen a partir de las tres perspectivas teóricas señaladas anteriormente.

Según el título de la mención encontramos la siguiente distribución (ver Tabla 4):

\begin{tabular}{|l|c|}
\hline \multicolumn{2}{|c|}{ TABLA 4. Títulos de mención por universidades } \\
\hline \multicolumn{1}{|c|}{ Título de la mención } & N. ${ }^{\circ}$ de universidades en las que se cursa \\
\hline Pedagogía Terapéutica & 10 \\
\hline Modelo Integrador: & 20 \\
\hline - Educación Especial & $(11)$ \\
\hline - Atención a la diversidad & $(5)$ \\
\hline - NEE & $(4)$ \\
\hline Modelo Inclusivo & 4 \\
\hline Sin mención específica & 6 \\
\hline
\end{tabular}

El total de menciones es de 34, porque la Universidad de Murcia ofrece dos menciones que podemos situar en el marco que nos ocupa. Señalar, también, que hay 6 universidades que no ofrecen ninguna mención específica relacionada con la temática abordada. El número total de créditos ECTS que conforman cada una de estas menciones presenta un amplio abanico, que va desde los 18 hasta los 48 créditos ECTS. Mostramos la carga lectiva de estas menciones agrupados de la siguiente forma:

- Menos de 30 créditos: 9 universidades.

- Entre 30 y 35 créditos: 12 universidades.

- Entre 36 y 40 créditos: 9 universidades.

- Más de 40 créditos: 4 universidades. 
Señalar que el peso total de créditos ECTS con mayor presencia es:

- 36 créditos: 6 universidades.

- 30 créditos: 9 universidades.

- 24 créditos: 4 universidades.

El resto se distribuye entre una o dos universidades por cada número de créditos ya indicados.

Si nos introducimos en la organización de estas menciones encontramos que el número de asignaturas teóricas que han de cursar los estudiantes para obtener la mención se distribuye según el gráfico siguiente (ver Figura 1). La mayoría de las universidades, diecisiete concretamente, apuesta por un total de 4 asignaturas para cursar la mención:

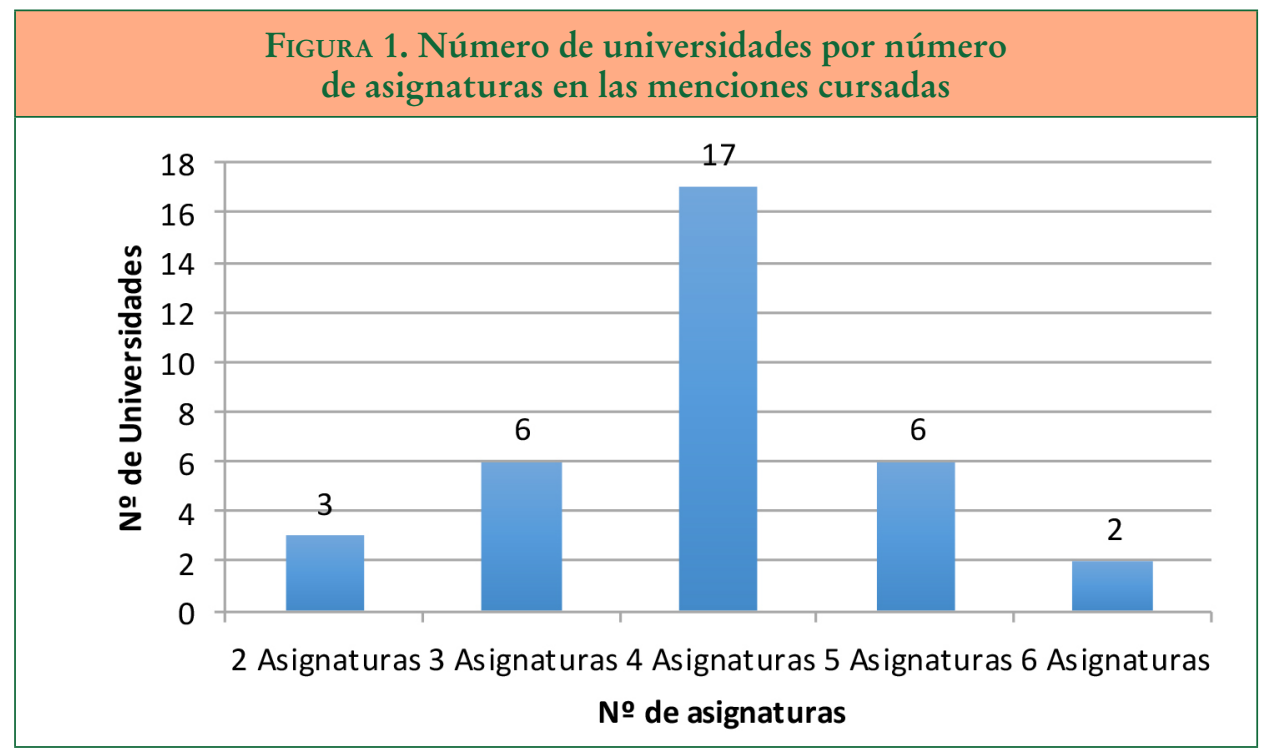

El número de créditos ECTS de estas asignaturas se distribuye de la siguiente manera: 


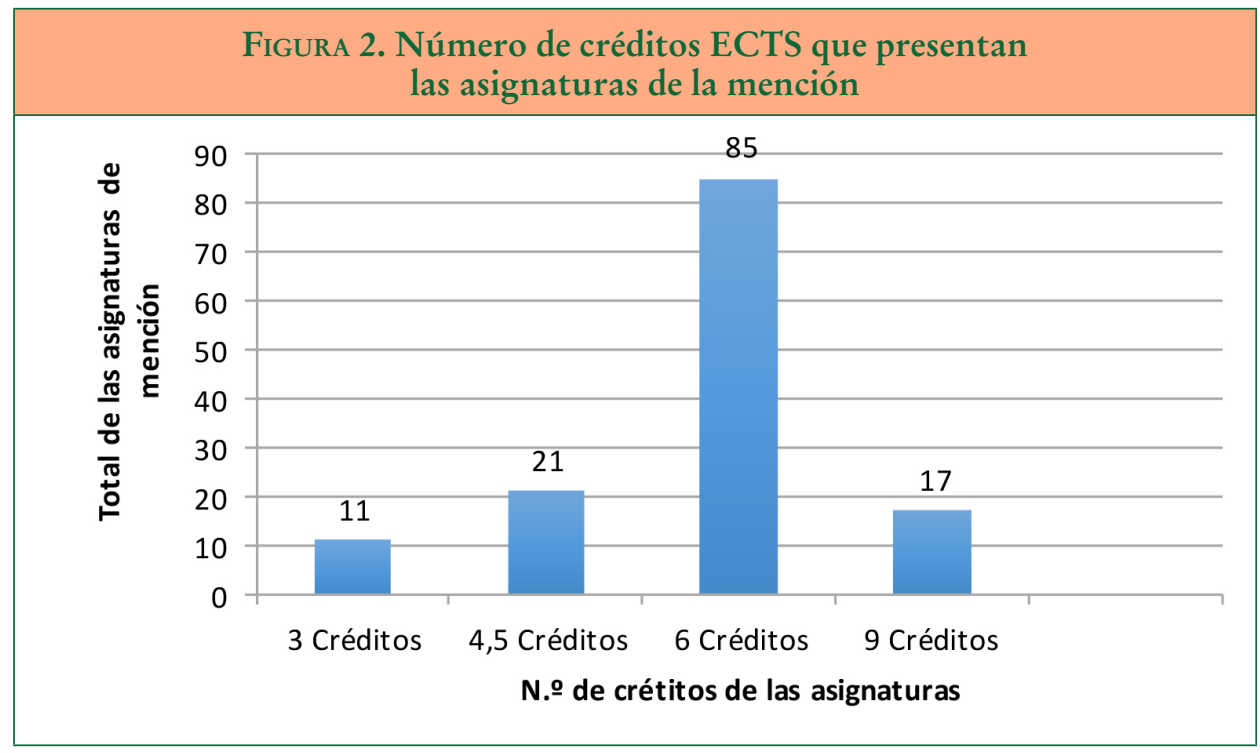

Merece destacar en el análisis de las menciones la inclusión de algunos créditos de prácticum específicos y propios de la mención: 13 universidades no lo contemplan en tanto que 20 universidades sí lo incluyen. Los créditos dedicados al prácticum de mención van desde los 6 hasta los 24 créditos, distribuidos de la manera siguiente:

- 12 universidades: de 9 a 12 créditos ECTS.

- 7 universidades: de 13 a 20 créditos ECTS.

- 1 universidad: más de 20 créditos ECTS.

Señalar que el número de créditos ECTS con mayor prevalencia es:

- 8 universidades: 12 créditos ECTS.

- 4 universidades: 15 créditos ECTS.

Por último, hay que señalar que solamente 4 universidades incluyen el TFG relacionado con la mención, con créditos que cubren 6,8 o 9 créditos.

\section{Discusión y conclusiones}

Si pretendemos conseguir un modelo educativo inclusivo, tal y como aconsejan y plantean todos los organismos internacionales que tratan, analizan y estudian la educación; si creemos que el modelo de educación inclusiva es el mejor que conocemos en la actualidad; si defendemos que la educación debe ser de calidad para todos los estudiantes sin excepción, donde la equidad sea un valor básico y clave, donde la ex- 
clusión, la marginación o la segregación de cualquier estudiante por cualquier motivo no figure en ninguna de las propuestas y modelos de actuación y decisión educativa, debemos potenciar y favorecer una formación inicial y permanente de todo el profesorado en actitudes, conocimientos, habilidades y destrezas inclusivas.

Partimos de la base de que la inclusión se fundamenta en dos pilares básicos: por una parte, la actitud, las creencias, los referentes teóricos y de pensamiento, que dirigen nuestra intervención y actuación en la escuela; por otra parte, los modelos de intervención didáctica, la organización escolar y la metodología que aplicamos en las aulas; es imprescindible incorporar estos elementos en la formación inicial de los maestros (Figueiredo y Ortiz, 2017; Martínez et al., 2019).

Podemos analizar los resultados presentados en este trabajo a partir de tres bloques complementarios y confluyentes en sus ideas y actuaciones: la fundamentación conceptual, que explica y justifica una postura y un modelo formativo; la formación general que reciben todos los futuros maestros y maestras de Educación Primaria, y, por último, la formación específica a recibir por los maestros y maestras de apoyo.

En cuanto a la fundamentación conceptual, para alcanzar el objetivo de una escuela para todos y todas se necesita, entre otras medidas, una adecuada formación de todos los y las docentes dirigida a un doble objetivo (León, 1999): conseguir la inclusión de todos los alumnos y alumnas en las aulas ordinarias y promover la colaboración y el trabajo en equipo entre todo el profesorado.

Esta formación debe salirse definitivamente del modelo médico, señala Chiner (2018), y dirigirse hacia un modelo social. Debe desarrollar y mejorar las competencias de todos los y las profesionales implicados en el proceso educativo para potenciar un modelo educativo plenamente inclusivo. Se necesita consolidar conocimientos básicos, cambiar actitudes y adoptar metodologías activas del aprendizaje.

$\mathrm{Al}$ analizar la naturaleza de las asignaturas incluidas en los diferentes planes de estudio, hemos constatado que la formación inicial actual sigue dominada por el paradigma de la integración y, en algunos casos, por el paradigma deficitario. No se ha producido, por lo general, en los planes de estudio analizados un cambio y una apuesta decidida por la implantación del paradigma inclusivo. No se refleja ni se percibe una implementación mayoritaria de los principios de la educación inclusiva, como escriben Vélez-Calvo et al. (2016, p. 89):

Pese a que el Plan Bolonia supuso ampliar a cuatro cursos de formación inicial de los futuros maestros, la educación inclusiva no es un asunto que haya ganado peso en los planes de estudio. De hecho, en algunos casos ha perdido importancia, lo que denota claramente que la formación del profesorado en educación inclusiva no ha sido ni mucho menos una prioridad para quienes han diseñado el currículo.

Paralelamente, se mantienen las propuestas formativas propias de tiempos pasados; mantenemos las nominaciones de Pedagogía Terapéutica o Educación Especial, propias de paradigmas ya superados, y no se ha producido el cambio hacia propuestas más inclusivas como la de profesor de apoyo. Hay un desfase significativo y muy llamativo entre las propuestas de la investigación y del conocimiento en educación 
con los planteamientos y propuestas formativas en el grado de Maestro en Educación Primaria. Los programas formativos analizados mantienen propuestas y títulos categorizados y fundamentados en los déficits del alumnado y asignaturas que se refieren a las distintas patologías o deficiencias, siempre centradas en las limitaciones o problemática de los alumnos. Todo ello pese a que la investigación sobre los principios y fundamentos de la educación inclusiva ha demostrado que el problema no es el individuo, sino el contexto: las oportunidades, actuaciones y propuestas realizadas desde los diferentes procesos de enseñanza y de aprendizaje. Hay una clara y preocupante brecha entre la bibliografía actual, situada en el paradigma inclusivo, como ejemplo, el concepto de barreras a la participación y al aprendizaje (Booth y Ainscow, 2002), y la oferta formativa, que sigue fijada en los paradigmas deficitario o integrador, que se centra en las necesidades del alumnado, como, por ejemplo, el concepto de necesidades educativas especiales o las discapacidades sensoriales, cognitivas o físicas.

Referente a la formación general y común para todos los maestros y maestras de primaria, destacar que, si la educación inclusiva va dirigida a todo el alumnado, sin excepción ni categorización, son todos los maestros los que deben recibir una formación amplia y en profundidad relacionada con las actitudes, destrezas y conocimientos propios de una educación para todos. Echeita (2012, p. 12) lo describe con claridad: "Refuerza el argumento de que una educación inclusiva es responsabilidad de todo el profesorado, sin excepciones, y que la preparación de todos los profesores/ as para trabajar en centros educativos inclusivos es responsabilidad de todos". Proponemos que esta formación cumpla con cuatro ideas claves:

a) No hay categorías de alumnos, pues la diversidad entre las personas es un hecho natural e incuestionable.

b) El problema de las dificultades de aprendizaje no está en el alumnado y sus características, sino en las oportunidades que se ofrecen desde el contexto en los procesos de enseñanza y de aprendizaje.

c) El agrupamiento de los alumnos debe ser siempre heterogéneo, puesto que debemos sustituir en nuestras decisiones la lógica de la homogeneidad, dominante actualmente en las escuelas, por la lógica de la heterogeneidad, que nos permite respetar, atender y aceptar las diferencias.

d) El profesorado debe trabajar en equipo, donde la colaboración presida las actuaciones y decisiones conjuntas entre tutores y profesorado de apoyo.

La formación común y obligatoria que se ofrece en los grados analizados es insuficiente en el ámbito de la educación inclusiva, puesto que se reduce a una o dos asignaturas de 6 créditos ECTS. Además, los planteamientos que se ofrecen en estas asignaturas, en su gran mayoría, siguen situados en términos ya superados del paradigma deficitario y/o integrador. Hay una significativa brecha entre las propuestas formativas y los principios y actuaciones de la educación inclusiva. Esta realidad dificulta e impide un acercamiento de la práctica educativa a postulados de una escuela para todos, porque los docentes no tienen la formación requerida para promover los cambios deseados para alcanzar definitivamente la aplicación del paradigma de la inclusión en los centros escolares. 
Nos sucede lo mismo en las leyes educativas, todos estamos de acuerdo y apostamos por la necesidad del cambio del modelo de la escuela selectiva, propia de tiempos pasados, al modelo inclusivo, como mejor respuesta y más adaptada a la realidad social actual, pero seguimos fijados y anclados en propuestas científicamente ya superadas. Es preciso y urgente perder el miedo al cambio y aplicar las perspectivas, contenidos y actuaciones propios de la escuela inclusiva.

Este planteamiento exige la incorporación de elementos de formación basada en los principios señalados para romper con la dicotomía propia de modelos deficitarios o integradores entre maestros generalistas y especialistas. León (1999) ya planteaba la necesidad de una formación polivalente y común de todo el profesorado que va a trabajar en la diversidad.

La necesidad de una formación donde se profundice y amplíen los contenidos, destrezas y habilidades en el ámbito de la educación inclusiva es una necesidad incuestionable ya que todos los maestros y maestras deben tener una relevante y potente formación al respeto.

La realidad actual nos obliga también a plantear la necesidad de ofrecer en la formación en el grado de Maestro en Educación Primaria una mención, especialidad o profundización en los temas de la educación inclusiva, que forme a los maestros y maestras de apoyo para contribuir a la construcción de los nuevos centros educativos que deseamos. Todo ello, por dos razones claves:

a) La formación ofrecida a todos los futuros maestros y maestras es claramente insuficiente y pobre en el ámbito de la educación inclusiva, como ya hemos analizado anteriormente.

b) El trabajo cooperativo, en igualdad, entre los docentes en un mismo espacio es imprescindible para atender de manera adecuada, en el marco de la inclusión, a toda la diversidad del alumnado. Se requiere de formaciones complementarias no para descargar de responsabilidad a ningún docente, sino para encajar las actuaciones de apoyo y mejora de la respuesta educativa.

Esta investigación pone de manifiesto que las menciones ofrecidas en el ámbito de la educación inclusiva, de las universidades analizadas, se mantienen en los parámetros del paradigma deficitario y/o integrador, tanto en los títulos como en la formación propuesta. Además, por su extensión y profundización son claramente poco relevantes y significativos. Es imprescindible una reflexión conjunta de todo el profesorado universitario que trabaja, investiga e imparte docencia en este ámbito para revolucionar los planteamientos de la oferta formativa actual y eliminar de los planes de estudio las temáticas y principios de paradigmas de conocimiento ya superados por la investigación. Es imprescindible apostar por la educación inclusiva, como única vía posible para alcanzar una educación para todos y todas, que aumente la justicia social, la cohesión y la equidad de todo el alumnado.

A modo de conclusión, y contextualizando el presente estudio en las universidades analizadas, presentamos ideas clave que pueden ser de gran utilidad para plantear futuras actuaciones y retos en la formación inicial de los maestros y maestras de Educación Primaria. 
1. La formación inicial actual de maestros de Educación Primaria en el ámbito de la educación inclusiva es claramente insuficiente.

2. La oferta formativa se mantiene, por regla general y mayoritaria, con planteamientos situados en el paradigma deficitario y/o integrador.

3. Se mantienen títulos de asignaturas y de menciones claramente superados por los conocimientos producidos por las investigaciones actuales y contenidos en una extensa bibliografía que, además, son incoherentes con los principios y las actuaciones de una escuela para todos.

4. Es preciso y urgente romper con el círculo vicioso actual entre mantener una propuesta formativa coherente con la nomenclatura utilizada por el Ministerio de Educación y apostar, desde la formación, de manera decidida y potente por el paradigma de la educación inclusiva, que elimine definitivamente los procesos, planteamientos e ideas que no aporten para la consecución de una verdadera y real escuela para todos.

5. La educación inclusiva es un modelo educativo para todo el alumnado, por ello todos los maestros deben recibir una formación coherente y suficiente en los planteamientos y actitudes del modelo de la educación inclusiva para responder de manera adecuada al reto de avanzar y conseguir una educación para todos y todas.

6. Esta formación debe centrarse en desarrollar modelos basados en la intervención, en la flexibilización de los procesos de enseñanza y de aprendizaje y no en categorizar al alumnado por sus características.

7. La evaluación de los conocimientos, procesos y, sobre todo, actitudes hacia la inclusión que se lleva a cabo en la formación inicial de los futuros maestros y maestras de Educación Primaria debe mejorar para llevar a cabo prácticas inclusivas reales en los centros escolares. Es imprescindible iniciar una reflexión para modificar los planes de estudio y posibilitar la implantación de una formación que desarrolle y permita aplicar las bases para alcanzar una educación para todos.

\section{Referencias bibliográficas}

Agencia Europea para el Desarrollo de la Educación Especial. (2003). Principios fundamentales de la educación de necesidades especiales. Recomendaciones para responsables politicos. Recuperado de https://www.european-agency.org/sites/default/files/key-principlesin-special-needs-education_keyp-es.pdf

Agencia Europea para el Desarrollo de la Educación Especial. (2011). Formación del profesorado para la educación inclusiva. Recuperado de https://www.european-agency. org/sites/default/files/te4i-synthesis-report-es.pdf

Alba, C. (2017). Diseño Universal para el Aprendizaje: educación para todos y prácticas de enseñanza inclusiva. Morata.

Arreaza, F. (2009). Inclusión y competencias básicas. En Actas I Congreso Nacional de Buenas Prácticas de Educación, Diversidad y Empleo. Gobierno de Murcia. 
Biencinto-López, Ch., González-Barbera, C., García-García, M., Sánchez-Delgado, P. y Madrid-Vivar, D. (2009). Diseño y propiedades psicométricas del Avaco-Evadie. Cuestionario para la evaluación de la atención a la diversidad como dimensión educativa en las instituciones escolares. Relieve, 15(1), 1-36. Recuperado de https:/www.uv.es/Relieve/ v15n1/RELIEVEv15n1_4.pdf

BOE. (2007). Orden Eci/3857/2007 de 27 de diciembre, por la que se establecen los requisitos para la verificación de los títulos universitarios oficiales que habilitan para el ejercicio de la profesión de Maestro en Educación Primaria, 312.

Bоотн, T. (2006). Manteniendo el futuro con vida; convirtiendo los valores de inclusión en acciones. En M. Á. Verdugo y B. Jordán de Urríes (Coords.), Rompiendo inercias. Claves para avanzar. VI Jornadas Cientificas de Investigación sobre Personas con Discapacidad (pp. 211-218). Amarú.

Booth, T. y Ainscow, M. (2002). Índice de inclusión. Desarrollando el aprendizaje y la participación en las escuelas. Orealc/Unesco.

Chiner, E. (2018). La educación inclusiva y su desarrollo en el marco legislativo español. International Studies on Law Education, 29(30), 53-66. Recuperado de http://rua.ua.es/dspace/ handle/10045/70322

EcheitA, G. (2012). Competencias esenciales en la formación inicial de un profesorado inclusivo. Un proyecto de la Agencia Europea para el Desarrollo de las Necesidades Educativas Especiales. Tendencias Pedagógicas, 19, 7-24. Recuperado de https://revistas.uam.es/tendenciaspedagogicas/article/view/1999

Escudero, J. M. y Martínez, B. (2011). Educación inclusiva y cambio escolar. Revista Iberoamericana de Educación, 55, 85-105. Recuperado de file://C:/Users/Windows/Downloads/rie 55a03\%20(3).pdf

Figueredo, V. y Ortiz, L. (2017). Formación inicial del profesorado para la inclusión de la diversidad cultural. Revista Digital de Investigación en Docencia Universitaria, 11 (1), 38-61. Recuperado de file:///C:/Users/Windows/Downloads/Dialnet-FormacionInicialDelprofesoradoparaLaInclusionDeLaD-6049179.pdf

García-Llamas, J. L. (2008). Aulas inclusivas. Bordón, 60(4), 89-105. Recuperado de https:// recyt.fecyt.es/index.php/BORDON/article/view/28871

GonzÁlez-Gil, F., Martín-Pastor, E. y Orgaz, B. (2017). ¿Están los futuros profesores formados en inclusión? Validación de un cuestionario de evaluación. Aula Abierta, 46, 33-40. Recuperado de https://www.unioviedo.es/reunido/index.php/AA/article/view/11985

LEÓN, M. J. (1999). La formación del profesorado para una escuela para todos. Un análisis de los planes de estudio del maestro especialista en educación primaria y en educación especial de las universidades españolas. Profesorado: Revista de Currículum y Formación del Profesorado, 3(2), 1-24. Recuperado de https://www.ugr.es/ recfpro/rev32ART1.pdf

LOCE. (2002). Ley Orgánica 10/2002, de 23 de diciembre, de Calidad de la Educación. Boletín Oficial del Estado, 307.

LOE. (2006). Ley Orgánica 2/2006, de 3 de mayo, de Educación. Boletín Oficial del Estado, 106.

LOGSE. (1990). Ley Orgánica 1/1990, de 3 de octubre, de Ordenación General del Sistema Educativo. Boletín Oficial del Estado, 238.

LOMCE. (2013). Ley Orgánica 8/2013, de 9 de diciembre, para la mejora de la calidad educativa. Boletín Oficial del Estado, 295. 
Martínez, R., Porto, M. y Garrido, C. F. (2019). Aulas de educación especial en España: análisis comparado. Siglo Cero, 50(3), 271, 89-120. http://dx.doi.org/10.14201/scero201950389120

Montobbio, E. (1995). El viaje del Sr. Down al mundo de los adultos. Mason.

Muntaner, J. J. (2009). Escuela y discapacidad intelectual. Eduforma.

Oliver, M. (1998). ¿Una sociología de la discapacidad o una sociología discapacitada? En L. BurTon (Ed.), Discapacidad y sociedad. Recuperado de http://www.bivipas.unal.edu.co/ handle/10720/645

Organización De Las Naciones Unidas. (2006). Convención sobre los Derechos de las Personas con Discapacidad. Recuperado de http://www.un.org/spanish/disabilities/default. asp?id $=497$

Organization For Economic Co-Operation And Development. (2012). Equity and quality in education: supporting disadvantaged students and schools. Recuperado de http://www. oecd.org/education/school/equityandqualityineducation-supportingdisadvantagedstudentsandschools.htm

Thomas, G. y Loxley, A. (2007). Deconstructing special education. Open University Press.

Torres, J. A. y Castillo, S. (2016). Incidencia de las políticas de apoyo educativo en las estructuras organizativas de los centros desde la perspectiva del profesorado: un estudio en la provincia de Jaén. Educación XXI, 19(2), 205-228. https://doi:10.5944/educxxi1.16462

United Nations Educational, Scientific And Cultural Organization. (1994). Declaración de Salamanca. Informe final. Conferencia Mundial sobre Necesidades Educativas Especiales: Acceso y Calidad. Ministerio de Educación y Ciencia. Secretaría de Estado de Educación.

United Nations Educational, Scientific And Cultural Organization. (2005). Guidelines for inclusion: ensuring access to education for all. Unesco

United Nations Educational, Scientific And Cultural Organization. (2008). La Educación Inclusiva: el camino hacia el futuro: conclusiones y recomendaciones de la $48 .{ }^{a}$ reunión de la Conferencia Internacional de Educación (CIE). Unesco.

United Nations Educational, Scientific And Cultural Organization. (2015). Declaración de Incheon: de la aprobación a la aplicación. Recuperado de https://unesdoc.unesco. org/ark:/48223/pf0000245656_spa

Vélez-Calvo, X., Tárraga-Mínguez, R., Fernández-Andrés, M. ${ }^{a}$ I. y Sanz-Cervera, P. (2016). Formación inicial de maestros en Educación Inclusiva: una comparación entre Ecuador y España. Revista Nacional e Internacional de Educación Inclusiva, 9(3), 75-94. Recuperado de https://dialnet.unirioja.es/servlet/articulo?codigo $=5986222$ 


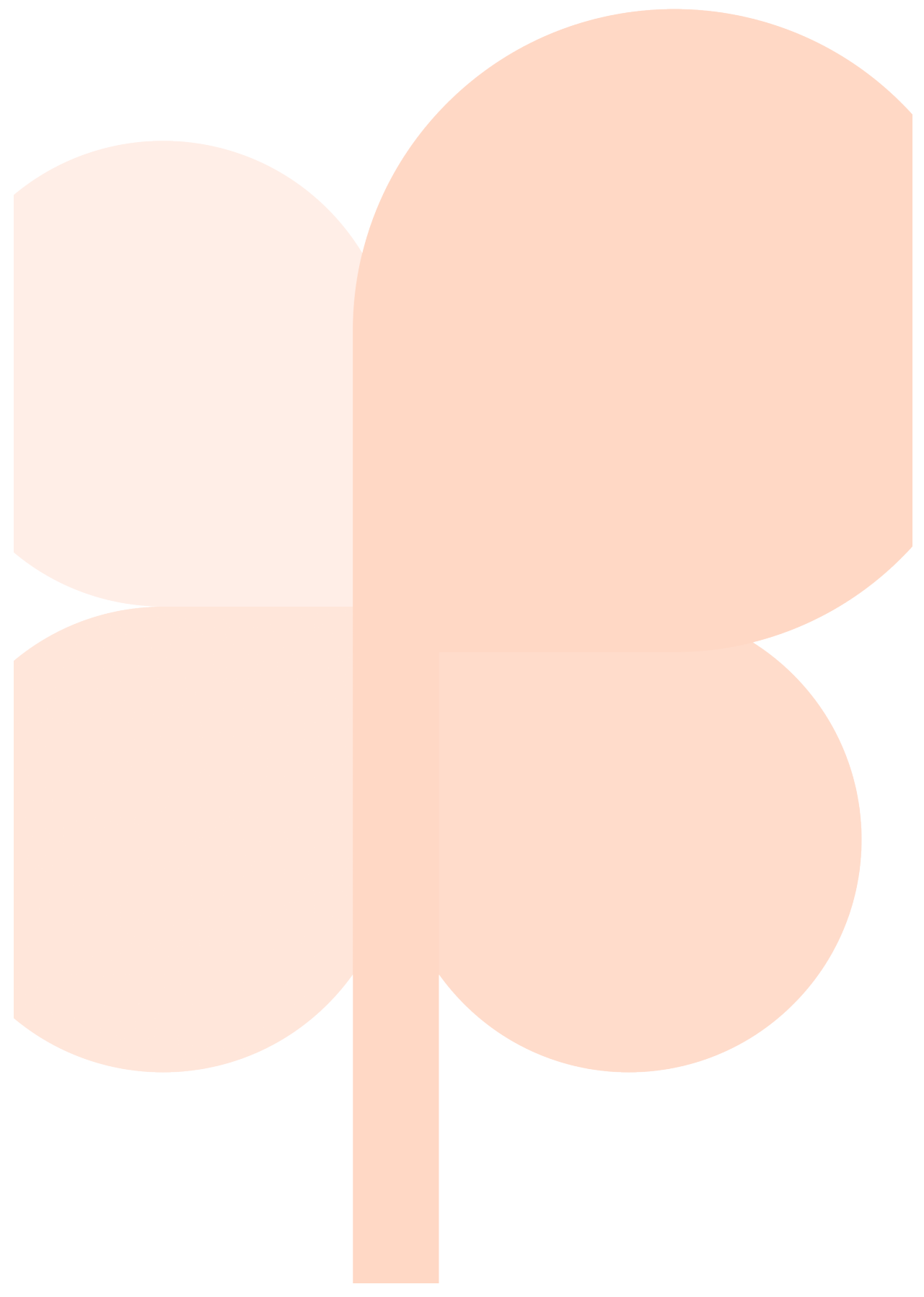

